\title{
Digital Technology in Movement Disorders: Updates, Applications, and Challenges
}

\author{
Jamie L. Adams ${ }^{1,2} \cdot$ Karlo J. Lizarraga ${ }^{1,2} \cdot$ Emma M. Waddell ${ }^{2} \cdot$ Taylor L. Myers $^{2}$ • Stella Jensen-Roberts ${ }^{2}$. \\ Joseph S. Modica ${ }^{1} \cdot$ Ruth B. Schneider ${ }^{1,2}$ (D)
}

Accepted: 21 January 2021 / Published online: 3 March 2021

(C) The Author(s), under exclusive licence to Springer Science+Business Media, LLC part of Springer Nature 2021

\begin{abstract}
Purpose of Review Digital technology affords the opportunity to provide objective, frequent, and sensitive assessment of disease outside of the clinic environment. This article reviews recent literature on the application of digital technology in movement disorders, with a focus on Parkinson's disease (PD) and Huntington's disease.

Recent Findings Recent research has demonstrated the ability for digital technology to discriminate between individuals with and without PD, identify those at high risk for PD, quantify specific motor features, predict clinical events in PD, inform clinical management, and generate novel insights.

Summary Digital technology has enormous potential to transform clinical research and care in movement disorders. However, more work is needed to better validate existing digital measures, including in new populations, and to develop new more holistic digital measures that move beyond motor features.
\end{abstract}

Keywords Digital technology $\cdot$ Smartphones $\cdot$ Wearable devices $\cdot$ Parkinson's disease $\cdot$ Huntington's disease $\cdot$ Movement disorders

\section{Introduction}

Movement disorders, such as Parkinson's disease, essential tremor, and Huntington's disease, are inherently complex, characterized by a wide array of motor and non-motor symptoms. This complexity, in combination with the lack of objective biomarkers of disease progression, creates a challenge in the assessment of disability and progression. Traditional rating scales are subjective, episodic, and typically limited to inperson visits. Traditional scales are thereby limited by rater variability, inadequate for the capture of fluctuations in symptoms, and unable to provide a comprehensive assessment. Additionally, the requirement for in-person visits is burdensome for individuals with neurodegenerative disorders, who are likely to have functional mobility impairments [1]. The

Ruth B. Schneider

Ruth_schneider@urmc.rochester.edu

1 Department of Neurology, University of Rochester, 265 Crittenden Blvd, Box MIND, Rochester, NY 14642, USA

2 Center for Health + Technology, University of Rochester, Rochester, NY, USA
COVID-19 pandemic has disrupted clinical practice, forcing a switch to telemedicine for the provision of clinical care [2]. The pandemic has also disrupted clinical research and raised appropriate concerns regarding participant safety [3, 4]. At least for the near term, clinical and research operations will need to adapt to this new environment. One critical adaptation will be the incorporation of alternative means of assessment and treatment outside of the clinic environment.

Digital tools, such as smartphones and wearable sensors, present an opportunity to objectively, frequently, and remotely assess individuals with movement disorders across different settings. In turn, this holds the promise of earlier identification of individuals at risk for or with disease, improved disease phenotyping, enhanced sensitivity for the detection of disease progression that may speed the development of new therapeutics, and improved clinical management. Emerging deep brain stimulation (DBS) systems illustrate some of the clinical applications of wearable technology and can directly measure electrophysiological activity, which can inform the development of remote programming and adaptive DBS systems.

Smartphones, equipped with accelerometers, gyroscopes, and global positioning (GPS) technology, are widely accessible and used by over 3 billion people worldwide on a daily 
basis [5]. Their increasing ubiquity makes them appealing digital tools for clinical care and research. Wearable devices may be equipped with a wider array of sensors, including electromyography, electrocardiography, temperature sensors, magnetometers, and more. Unlike smartphones, wearable devices generally do not require active participant engagement and can be worn in several different locations. This presents additional advantages, such as the ability to detect rare events, capture performance of daily activities, more extensively characterize disease features, and more readily capture non-motor features like sleep. Wearable sensor and smartphone studies have proven to be feasible, even in large cohorts, and are well accepted by patients and research participants [6-9]. Here we review the recent literature on applications of smartphones and wearable sensors in movement disorders as well as the development of adaptive DBS systems. While important, this review does not cover multiple relevant topics, including regulatory barriers $[10,11]$, fitness trackers $[12,13]$, alternative forms of passive home monitoring [14], integration of digital platforms [10], and analytical methods.

\section{Parkinson's Disease}

Studies evaluating smartphones and wearable sensors in PD are the most abundant. Multiple studies have focused on the ability to discriminate between individuals with PD and those without PD. For example, data from the mPower application (Sage Bionetworks, Seattle, Washington) walking task [15] and tapping task [16] and the Roche PD Mobile application (Roche, Basel, Switzerland) sustained phonation, rest tremor, postural tremor, tapping, balance, and gait tasks [17••] can accurately distinguish between individuals with PD and healthy controls (Table 1). Several studies have shown that wearable devices can distinguish between individuals with PD (both untreated and treated) and healthy controls [18] as well as different types of parkinsonism and dementia [19, 20]. Smartphones can be used to discriminate between essential tremor and PD [21]. One wearable sensor study used wristworn accelerometer devices to develop a tremor stability index, which can be used to distinguish between essential tremor and PD tremor with high specificity, sensitivity, and accuracy [22] (Table 2). While digital technology cannot and should not replace clinical diagnosis, this type of diagnostic aid could be useful, for example, in cases where tremor classification is difficult to determine by clinical observation.

Beyond their potential use in confirming the diagnosis of $\mathrm{PD}$, digital technologies offer the potential to enable identification of prodromal PD and evaluate risk for phenoconversion to manifest PD. Individuals at high risk for development of PD - such as those with LRRK2 or other genetic mutations, hyposmia, or idiopathic REM sleep behavior disorder (iRBD), in particular-may benefit from earlier identification of PD signs. Among those with iRBD, motor symptoms and subtle changes in motor examination have been shown to occur several years before phenoconversion [23]. Digital tools may be able to detect subtle motor changes even earlier than clinical examination. Relying on the performance of a series of active tasks, smartphone applications can accurately distinguish between healthy controls, individuals with PD, and individuals with iRBD with mean sensitivity and specificity ranging from 84.6 to $91.9 \%$ [24•]. In discriminating between healthy controls and iRBD, voice was the most salient factor [24•]. Consistent with this finding, smartphone speech analysis alone can accurately discriminate between individuals with PD and healthy controls $[25,26]$ and may be a promising feature for identifying those with iRBD who are at high risk for a neurodegenerative disease [27].

Del Din et al. evaluated a wearable device in nearly 700 healthy controls aged 50 to 80 years and found that differences in objective gait metrics between those who develop PD and those who do not could be detected about 4 years prior to diagnosis, suggesting that objective gait measures can help identify individuals with prodromal PD [28•]. Another study of older adults $(n=683)$ demonstrated that objective mobility measures obtained from a wearable sensor positioned on the back could predict development of parkinsonism [29]. However, not all features may be amenable to earlier detection; one study was unable to distinguish between individuals with early motor changes and healthy controls using smartphone finger tapping performance [30]. Appropriately targeted wearable devices may serve as digital biomarkers in $\mathrm{PD}$, enhancing identification of those with prodromal PD who may be eligible for participation in disease-modifying clinical trials and enhancing measurement of disease progression among those at high risk for PD.

Smartphones and wearable devices are commonly used to measure specific motor constructs. They can detect or quantify multiple motor symptoms in PD, including gait, falls, tremor, bradykinesia, motor fluctuations, and dyskinesias. For example, smartphone applications have been used to assess gait variability [31], assess postural sway [32], detect freezing of gait [33], identify postural instability [34], and assess sit-tostand transitions and turns in PD [17••]. Similarly, several studies have evaluated the use of wearable devices for assessing gait [35], detecting freezing of gait [36], and analyzing turning [37]. Of particular benefit in PD is the detection of falls and the evaluation of fall risk. Wearable devices can provide an objective and accurate measure of falls. Silva de Lima et al. evaluated falls using a wearable sensor in 2063 individuals with PD and 2063 matched controls and found that the sensors could accurately detect falls, which were nearly twice as frequent in those with PD as controls [38]. While a history of prior falls is a good predictor of future falls, fall prediction among those who have never fallen is more difficult [39]. Lo et al. demonstrated that a single performance of a 


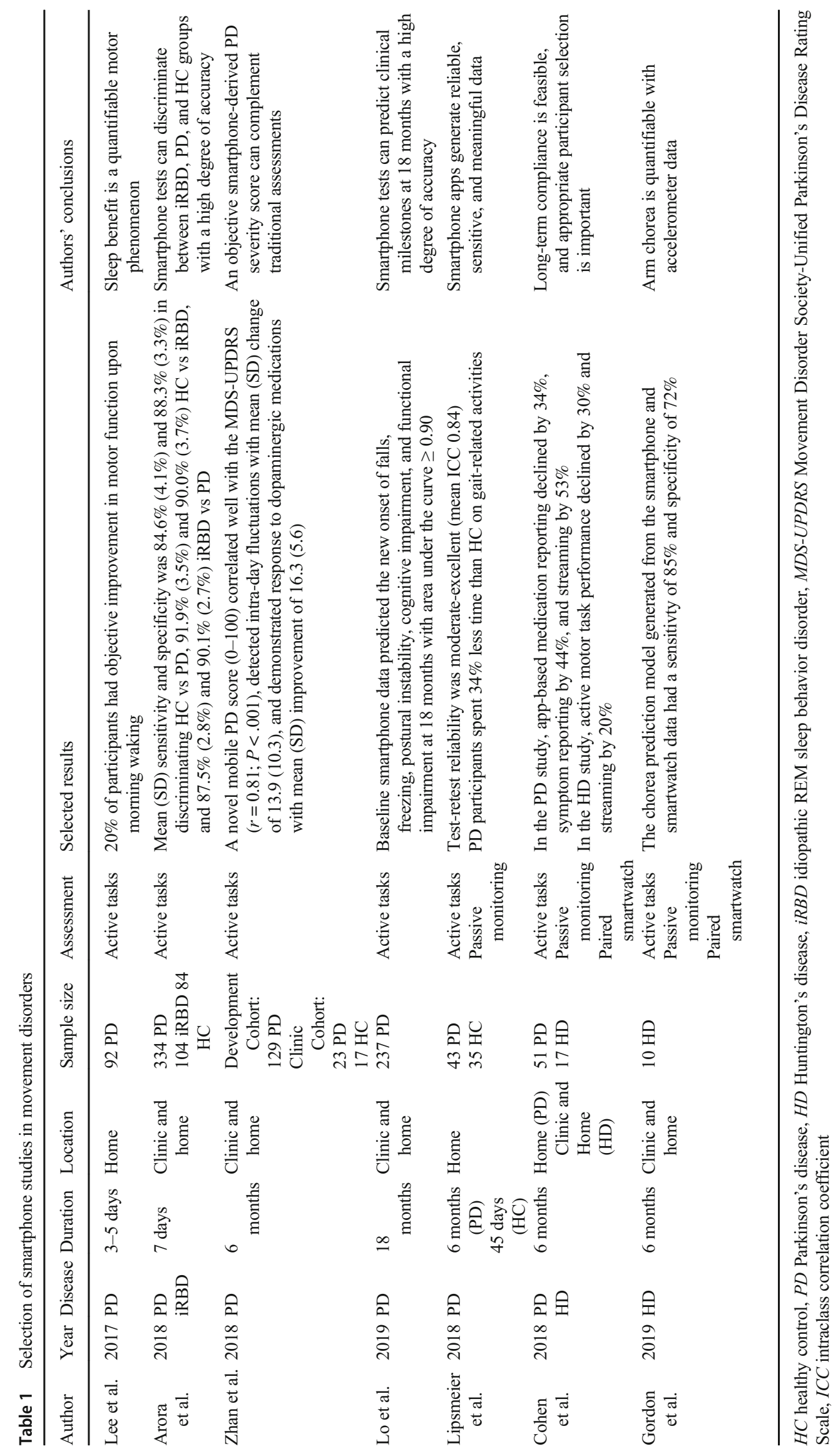




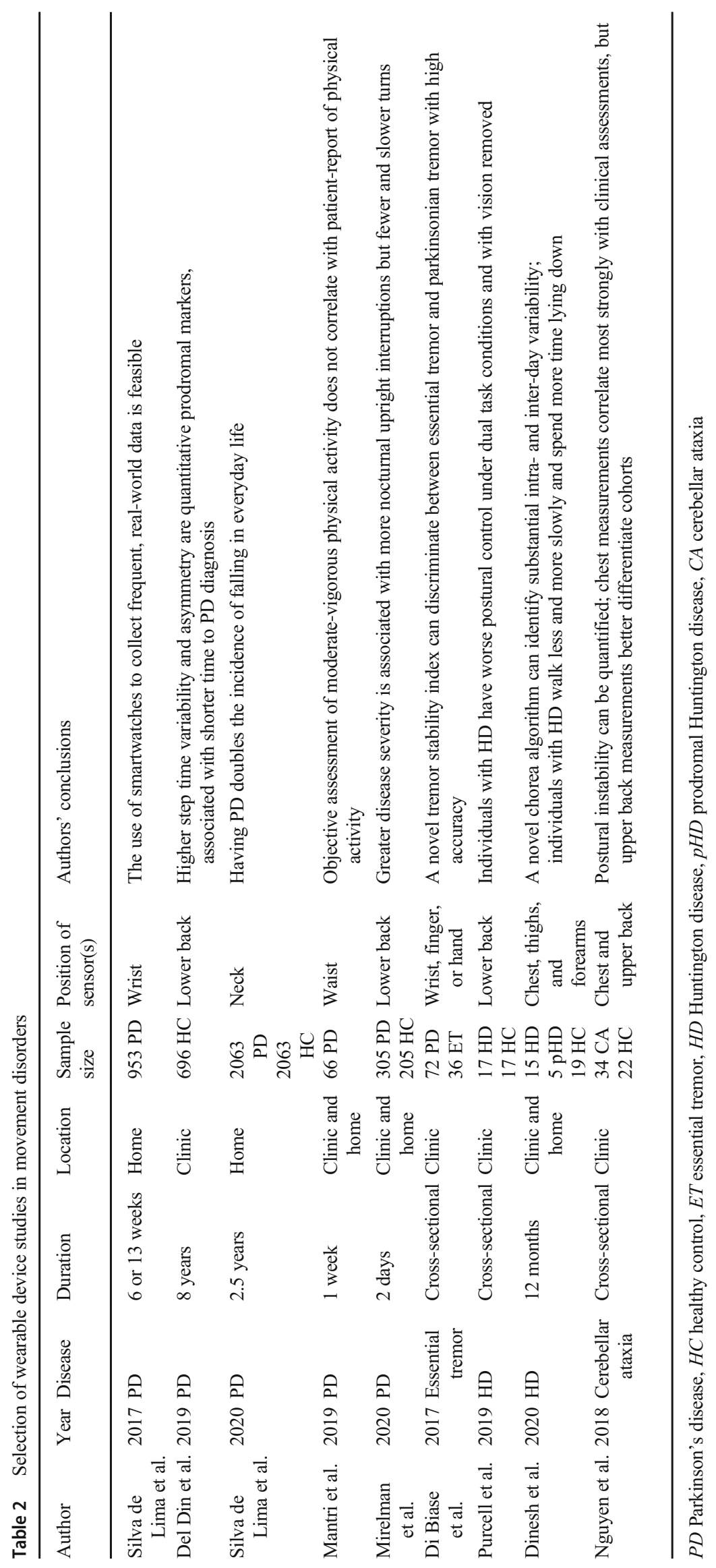


set of smartphone tasks could predict future development of falls, freezing of gait, and postural instability with a high degree of accuracy [40 ${ }^{\bullet}$. Thus, digital tools have the potential to aid in evaluation of multiple different gait parameters and in clinical prognostication. Tremor has also been studied and quantified with both smartphone applications and wrist-worn devices, such as smartwatches [41-43]. Bradykinesia can similarly be quantified using smartphone applications [44, 45].

Lipsmeier et al. demonstrated the feasibility of incorporating smartphone-based active and passive assessments into PD clinical trials and the superior sensitivity of such assessments, which were able to detect motor abnormalities not captured on traditional motor scales [17••]. However, a 2018 review determined that fewer than $3 \%$ of on-going clinical trials in neurodegenerative diseases included a technology-based outcome measure [46]. To develop clinically meaningful outcome measures, we need to move beyond the assessment of isolated constructs towards more holistic and global assessments [10]. Performance on a number of smartphone active motor tasks can be used to construct a mobile PD score that demonstrates strong correlation with standard clinical measurements, captures intra-day fluctuations, and is responsive to medication intake [47]. This study demonstrates the potential for generating more global measures; however, more work is needed to move beyond assessment of motor features.

Wearable sensors offer clear advantages over smartphones with regard to passive data collection. For example, physical activity and sleep can be objectively assessed. Objective measurements of activity may differ from patient reports, as shown in the Mantri et al. study of veterans with $\mathrm{PD}$, which found that moderate-vigorous physical activity as measured by an Actigraph was infrequent in the cohort and did not correlate with self-report [48]. Another study found that traditional measures of PD do not reflect daily activity and that wearable devices can provide a more objective and complete assessment [49]. Mirelman et al. demonstrated the ability of wearable accelerometers to detect sleep interruptions and sleep turns and identified differences between individuals with advanced $\mathrm{PD}$, early $\mathrm{PD}$, and without $\mathrm{PD}\left[50^{\bullet}\right]$. This type of measure could be helpful in identifying individuals with early PD, serve as an objective measure of disease severity, and help with medication management. Smartphones can be used for some forms of passive data collection. For example, analysis of smartphone touchscreen typing can discriminate between individuals with early PD and controls [51-53]. GPS-enabled smartphone passive data collection can facilitate mapping of lifespace [54], which is one's geographic area of movement, and quantification of the frequency and duration of trips outside of the home. Such an approach has been used to demonstrate post-deep brain stimulation (DBS) improvements [55] and is appealing as a potentially clinically meaningful outcome measure.

Smartphones and wearable sensors can also inform clinical management, providing frequent, in-home data on a granular scale. Such an approach may be of particular benefit in the management of motor fluctuations. Integrated systems, such as PD_Manager [56], which enables passive and active data collection through a combination of a smartphone application, a smartwatch, and sensor insoles, are feasible for short-term use in individuals with moderate PD with motor fluctuations [43]. Another study demonstrated that an algorithm developed from a wrist and ankle sensor can objectively and accurately detect medication ON and OFF states [57]. These types of measures could serve as substitutes for cumbersome and subjective patient diaries, and efforts are underway by the Movement Disorders Society to create an e-Diary [58]. Smartphones can be used to monitor medication response [59] and assess diurnal variation [60]. Similarly, wearable sensors allow for remote titration of medications. For example, one study found that rotigotine dose changes and increases were higher in the group monitored by wearable devices compared to the group that was not monitored by wearable devices [61].

However, the incorporation of digital tools into clinical practice poses some challenges. Digital data must be presented in a manner that is informative and useful to clinicians. In one study, a wrist-worn device was used by 63 individuals with PD and provided useful information to guide treatment plans for $50(79 \%)$ of these individuals [62]. In another study, a smartwatch was paired with a smartphone application, and clinicians were involved in the iterative development of a clinician dashboard that could be used to inform changes in management [63]. Clinicians rated medication adherence and patientreported outcomes as the most informative and sensorderived measures as the least informative. This suggests that there is much progress to be made in the development of clinician-friendly data displays.

Digital tools also show promise for self-management and greater precision in treatment. Smartphone-based applications can provide access to educational resources, be used for selftracking and medication adjustments [64], and may improve short-term medication adherence [65]. Yet, in a randomized controlled trial, PD participants randomized to use a smartphone exercise application did not demonstrate any improvements in gait, speech, or dexterity compared with the control group [66], suggesting that simply providing access to such applications is insufficient. A biofeedback system combining a smartphone application and sensor-equipped belt for balance training in PD has been shown to be feasible [67]. More work is needed to determine whether such devices improve outcomes. 


\section{Other Movement Disorders}

Beyond PD, smartphone applications have been used to characterize orthostatic tremor [68], characterize orthostatic tremor-associated gait and balance impairment [69], assess gait impairment in drug-induced parkinsonism [70], and quantify Huntington's disease (HD) chorea $[71,72]$. Wearable sensors have been used even more broadly.

Wearable sensors are gaining traction in the evaluation of HD. Several studies have evaluated gait, posture, activity, balance, and falls in individuals with HD. One study measured postural sway in clinic and showed that individuals with HD have worse postural control during manipulated stance, vision, proprioception, and/or cognitive demand as compared with controls [73]. Another study showed that wearable iPod sensors equipped with a biofeedback app were able to measure and monitor trunk stability in individuals with HD and use this information for rehabilitative purposes [74]. Dinesh et al. developed a chorea algorithm and showed that chorea had substantial intra-day variability but minimal long-term change over the course of 12 months. This same study found that individuals with HD spent over half their day lying down, which was significantly different from individuals with $\mathrm{PD}$, prodromal $\mathrm{HD}$, or control participants [75].

Not surprisingly, wearable sensor studies in ataxia have focused on gait, balance, and fall risk. One study successfully used wearable sensors to objectively assess postural instability in patients with cerebellar ataxia; the authors noted that while clinical assessments could provide general trends, more quantitative assessment of balance dysfunction in this population was needed [76]. Another study found that step length variation as detected by sensors correlated with number of falls and disease severity [77]. Detection of increased fall risk with wearable sensors presents an opportunity to intervene earlier with assistive walking devices and physical therapy.

Wearable devices, mainly wrist-worn sensors, have also been used in essential tremor (ET). Smartwatches are able to quantify tremor, have shown good correlation between clinical scales, and are well-accepted by patients [78]. One study showed that prolonged and continuous monitoring in patients with ET with a smartwatch was feasible and revealed significant intra-day variability in tremor severity [79]. This variability has the potential to affect clinical trial outcomes, which are often based on brief snapshots of an individual's disease. Wearable devices can help overcome this challenge by enabling continuous and remote monitoring. Moreover, many people now personally own a smartwatch, which may allow for broad use of such devices in tremor research.

\section{Deep Brain Stimulation and Digital Technology}

Deep brain stimulation (DBS) is effective for the management of essential tremor (ET), dystonia, motor complications, and medication refractory tremor in PD [80-83]. Candidate selection can be difficult, and outcomes are variable and limited by both surgical placement and non-standardized programming techniques. Digital tools, including smartphones and wearable devices, may aid in the appropriate identification of DBS candidates [84]; pre-, intra-, and post-operative assessment of response to DBS adjustments [85-87]; and remote DBS programming. For instance, assessments of tremor, rigidity, and bradykinesia could be informed by the use of wearable sensors during awake DBS surgeries for PD or ET [88].

Recent innovations in DBS technology include novel leads that allow for directional and multi-target stimulation, as well as implantable pulse generators (IPG) and leads able to provide electrophysiological data that inform conventional DBS programming. The latter are able to record long-term electrophysiological information from the corresponding anatomical target, which can be correlated with symptoms and medications to optimize DBS programming during in-person visits. Such innovations will also permit adaptive DBS and remote programming to further improve outcomes in the near future. As opposed to data obtained from smartphones, sensors, and other wearable technology, directly measured pathophysiological brain activity could be used as surrogate biomarker of disease activity, progression, and response to treatment.

Self-adapting, closed-loop DBS technologies informed by real-time electrophysiological signals are currently under development. Most experimental adaptive DBS systems have used sub-thalamic nucleus (STN) local field potentials (LFP), particularly beta band signals, as the primary feedback mechanism in PD. Other electrophysiological techniques such as electrocorticography are also being explored $[89,90]$. Early clinical studies of adaptive DBS had relatively short duration and used externalized equipment in specialized laboratories. The initial adaptive approach to STN-DBS consisted of stopping DBS after achieving pathological STN beta signal suppression and resulted in similar or improved symptom control with less battery consumption when compared with conventional DBS [91-93]. Additionally, fewer stimulation-induced side effects may be associated with selective short beta burst suppression by adaptive DBS compared with conventional DBS [94]. In one study comparing 2 -h periods of conventional and adaptive STN-DBS in 10 patients with PD, adaptive DBS was associated with significantly reduced DBS energy delivered and improved dyskinesia control [95]. Case series in dystonia [96] and case reports in Tourette syndrome [97] provide evidence that electrophysiological signals recorded at single or multiple targets may be useful for adaptive DBS systems in other movement disorders. 


\section{Challenges and Future Directions}

\section{Need for Appropriate Validation of the Best Measures}

Despite the many advantages and opportunities for digital tools, there remain challenges in the use of these devices for clinical research and care in movement disorders. The amount of data that can be gathered by digital devices is immense and can be difficult to meaningfully condense or interpret. In particular, data collected in unsupervised settings can be difficult to validate $[98,99]$. Many initial validation studies are conducted in artificial clinic settings, bringing into question the value of data collected in the unsupervised, home setting. Comparison to "gold standard" traditional assessments is often sought, but given the subjective nature of these scales and the potential for digital measures to capture novel information, the meaning and value of these comparisons is unclear. Although non-motor symptoms, such as anxiety, depression, fatigue, and cognitive impairment, are important drivers of functional impairment and health-related quality of life in PD [100-102], most studies have focused on the measurement of motor symptoms [46]. Digital outcomes that provide more global assessments of function are needed.

\section{Limitations of Smartphones}

Part of the appeal of smartphones is their increasing ubiquity, but smartphone ownership varies substantially between and within countries, and younger, wealthier individuals with higher levels of education are more likely to own one [103, 104]. Relying on patients or research participants to provide their own smartphone will exacerbate the existing "digital divide" [105]. Most smartphone studies have focused on the performance of active tasks, such as finger tapping, which can be conducted frequently and in natural environments but may not be meaningful to patients. Moreover, smartphones are less well equipped to capture performance of more natural, day-today activities that may be more meaningful to patients.

\section{Challenges to Long-term, Consistent Use of Smartphone Applications}

Studies have consistently demonstrated that compliance with smartphone applications diminishes over time. Nearly 10,000 participants participated in the original mPower application study and consented to broad sharing of their data; however, fewer than $10 \%$ contributed data on a minimum of 5 days over a 6-month period [9]. Similarly, in a study pairing a smartphone with a smartwatch, use declined steadily over the 6-month study period, and nearly a quarter of participants failed to complete the study [63]. Specifically, medication reporting declined by $34 \%$, symptom reporting by $44 \%$, and smartwatch streaming by 53\% [106]. In a 100-day study examining a different app, only a third of participants completed a health-rated quality of life questionnaire at baseline and end of study [107]. Our own experience has been consistent with these reports. In an ongoing long-term remote follow-up study of phase 3 clinical trial participants, participants are asked to complete active smartphone tasks daily for 2 weeks every quarter [108]. The first 2 -week period saw a compliance rate of $61 \%$ that dropped to $14 \%$ in the second quarter and has averaged out to $22 \%$ across all quarters completed thus far. More work is needed to improve the "stickiness" of smartphone applications. Approaches may include sophisticated tracking and display of individual performance in the short- and long-term, improved notification systems, creation of a digital community of users, incorporation of educational materials, and the ability to readily share personal data with your clinician.

\section{Limitations of Wearable Sensors}

There are numerous consumer- and research-grade wearable devices on the market with variable sampling rates and algorithms, making comparisons difficult and limiting generalizability of results. Furthermore, the refinement of simple and reliable algorithms that can monitor more complex movements like dyskinesias or chorea is needed. While most devices have proven to be well-accepted and feasible to use in patients with neurodegenerative disease, application and use of devices becomes more challenging, particularly in the home setting and over longer periods of time. In our own experience, most participants are enthusiastic about engaging with wearable sensors, but many have little to no previous experience with such technology. As such, they face a steep learning curve and are often heavily reliant on research coordinators for technology setup and troubleshooting. A care partner can be particularly helpful in managing these barriers, but prolonged difficulty can lead to discouragement and frustration. Internet access and speed are other potential obstacles to effective technology use, and streaming compliance may be an additional issue with some devices. In a feasibility study evaluating a smartwatch-smartphone system in two different cohorts, data streaming declined by $23 \%$ in the 13 -week cohort and 27\% in the 6-week cohort [7]. Additionally, researchgrade wearable devices may be prohibitively expensive, and the safety of some wearable devices with implantable systems (including DBS) has not been fully evaluated, limiting their utility.

\section{Challenges with Fully Adaptive DBS Systems}

Preliminary observations with fully adaptive DBS systems have reported issues with limited battery life, relatively low storage capacity, and obscuration of low-amplitude LFPs by significant artifact. Furthermore, electrophysiological data 
recorded from the same DBS contact that delivers electricity would pose additional technical problems such as low-quality signals $[91,95,109]$. A rechargeable IPG would address battery life, but other problems need to be solved before adaptive systems are ready for clinical use $[110,111]$. Additional electrocorticography might be safe and would have several advantages in terms of signal-to-noise ratio and independent data obtained from different parts of the motor circuitry [111, 112]. The ideal adaptive DBS system would simultaneously differentiate symptom specific from normal physiological signals and use this information in real-time to dynamically modify DBS settings.

\section{Conclusions}

Digital technology provides a means to objectively, frequently, and remotely assess multiple different facets of movement disorders in a natural environment. Smartphones and wearable devices may enable the earlier identification of individuals at risk for or with disease and may be more sensitive to disease progression, both of which may facilitate the identification of diseasemodifying treatments. Devices can provide new insights into disability and progression that complement standard clinical assessments and enable deep clinical phenotyping of neurodegenerative diseases [113]. It is likely that some combination of clinical scales, imaging, biosamples, and digital tools will be the best and most comprehensive way to characterize and monitor disease. Smartphone and wearable devices may enable more personalized treatment and improved clinical management. Directly measuring neurophysiological brain activity with implanted DBS systems could provide additional pathophysiological information to be used as surrogate biomarker of disease activity, progression, and response to treatment. Remote DBS programming and adaptive, closed-loop DBS systems that enable real-time modifications in settings based on this information are being explored.

However, better validation of new digital outcomes and tools is needed. Future research should prioritize (1) larger sample sizes with longer remote monitoring periods and longer follow-up; (2) assessment of new populations, including those with prodromal disease and more advanced disease; (3) comparison with patient-reported outcomes, (4) digital device data standardization and the development of data sharing platforms to enable cross-study comparisons; and (5) the assessment of more non-motor features towards the development of more holistic disease characterization. Nonetheless, we believe that digital tools hold enormous potential for improving care, research, and outcomes in movement disorders.

\section{Declarations}

Conflict of Interest The authors declare that they have no conflict of interest except for Dr. Ruth B. Schneider.

Dr. Ruth B. Schneider reports grants from the National Institutes of Health, grants from Acadia Pharmaceuticals, grants from Biohaven Pharmaceuticals, grants from The Michael J. Fox Foundation for Parkinson's Research, grants from CHDI Foundation, grants from Canadian Institute of Health Research, grants from Teva Pharmaceuticals, grants from Pfizer, and grants from Nuredis, Inc., outside the submitted work.

Human and Animal Rights and Informed Consent This article does not contain any studies with human or animal subjects performed by any of the authors.

\section{References}

Papers of particular interest, published recently, have been highlighted as:

- Of importance

•. Of major importance

1. Bouca-Machado R, Maetzler W, Ferreira JJ. What is functional mobility applied to Parkinson's disease? J Parkinsons Dis. 2018;8:121-30.

2. Grossman SN, Han SC, Balcer LJ, Kurzweil A, Weinberg H, Galetta SL, et al. Rapid implementation of virtual neurology in response to the COVID-19 pandemic. Neurology. 2020;94:1077-87.

3. Padala PR, Jendro AM, Gauss CH, Orr LC, Dean KT, Wilson KB, et al. Participant and caregiver perspectives on clinical research during Covid-19 pandemic. J Am Geriatr Soc. 2020;68:E14-8.

4. Papa SM, Brundin P, Fung VSC, Kang UJ, Burn DJ, Colosimo C, et al. Impact of the COVID-19 pandemic on Parkinson's disease and movement disorders. Mov Disord. 2020;35:711-5.

5. Newzoo, Number of smartphone users worldwide from 2016 to 2021,Newzoo, https://www.statista.com/statistics/330695/numberof-smartphone-users-worldwide/,

6. Botros A, Schütz N, Camenzind M, Urwyler P, Bolliger D, Vanbellingen T, et al. Long-term home-monitoring sensor technology in patients with Parkinson's disease-acceptance and adherence. Sensors (Basel, Switzerland). 2019;19:5169.

7. Silva de Lima AL, Hahn T, Evers LJW, de Vries NM, Cohen E, Afek $\mathrm{M}$, et al. Feasibility of large-scale deployment of multiple wearable sensors in Parkinson's disease. PLoS One. 2017;12:e189161.

8. Lee W, Evans A, Williams DR. Validation of a smartphone application measuring motor function in Parkinson's disease. J Parkinsons Dis. 2016;6:371-82.

9. Bot BM, Suver C, Neto EC, Kellen M, Klein A, Bare C, et al. The mPower study, Parkinson disease mobile data collected using ResearchKit. Sci Data. 2016;3:160011.

10. Espay AJ, Hausdorff JM, Sanchez-Ferro A, Klucken J, Merola A, Bonato $\mathrm{P}$, et al. A roadmap for implementation of patient-centered digital outcome measures in Parkinson's disease obtained using mobile health technologies. Mov Disord. 2019;34:657-63.

11. Badawy R, Hameed F, Bataille L, Little MA, Claes K, Saria S, et al. Metadata concepts for advancing the use of digital health technologies in clinical research. Digit Biomark. 2019;3:116-32.

12. Lamont RM, Daniel HL, Payne CL, Brauer SG. Accuracy of wearable physical activity trackers in people with Parkinson's disease. Gait Posture. 2018;63:104-8. 
13. Pradhan S, Kelly VE. Quantifying physical activity in early Parkinson disease using a commercial activity monitor. Parkinsonism Relat Disord. 2019;66:171-5.

14. Kabelac Z, Tarolli CG, Snyder C, Feldman B, Glidden A, Hsu CY, et al. Passive monitoring at home: a pilot study in Parkinson disease. Digit Biomark. 2019;3:22-30.

15. Mehrang S, Jauhiainen M, Pietil J, Puustinen J, Ruokolainen J, Nieminen H. Identification of Parkinson's disease utilizing a single self-recorded 20-step walking test acquired by smartphone's inertial measurement unit. Conf Proc IEEE Eng Med Biol Soc. 2018;2018: 2913-6.

16. Prince J, de Vos M. A deep learning framework for the remote detection of Parkinson's disease using smart-phone sensor data. Conf Proc IEEE Eng Med Biol Soc. 2018;2018:3144-7.

17.• Lipsmeier F, Taylor KI, Kilchenmann T, Wolf D, Scotland A, Schjodt-Eriksen J, et al. Evaluation of smartphone-based testing to generate exploratory outcome measures in a phase 1 Parkinson's disease clinical trial. Mov Disord. 2018:33:1287-97 The authors describes the deployment of a smartphone application in a PD clinical trial and demonstrate the test-retest reliability and sensitivity of smartphone assessments, their ability to discriminate between PD and healthy controls, and the potential to generate novel insights.

18. Di Lazzaro G, Ricci M, Al-Wardat M, Schirinzi T, Scalise S, Giannini F, et al. Technology-based objective measures detect subclinical axial signs in untreated, de novo Parkinson's disease. J Parkinsons Dis. 2020;10:113-22.

19. Mc Ardle R, Del Din S, Galna B, Thomas A, Rochester L. Differentiating dementia disease subtypes with gait analysis: feasibility of wearable sensors? Gait Posture. 2020;76:372-6.

20. De Vos M, Prince J, Buchanan T, FitzGerald JJ, Antoniades CA. Discriminating progressive supranuclear palsy from Parkinson's disease using wearable technology and machine learning. Gait Posture. 2020;77:257-63.

21. Barrantes S, Sanchez Egea AJ, Gonzalez Rojas HA, Marti MJ, Compta Y, Valldeoriola F, et al. Differential diagnosis between Parkinson's disease and essential tremor using the smartphone's accelerometer. PLoS One. 2017;12:e0183843.

22. di Biase L, Brittain J-S, Shah SA, Pedrosa DJ, Cagnan H, Mathy A, Chen CC, Martín-Rodríguez JF, Mir P, Timmerman L, Schwingenschuh P, Bhatia K, Di Lazzaro V, Brown P (2017) Tremor stability index: a new tool for differential diagnosis in tremor syndromes. Brain: a journal of neurology 140, 1977-1986.

23. Fereshtehnejad SM, Yao C, Pelletier A, Montplaisir JY, Gagnon JF, Postuma RB. Evolution of prodromal Parkinson's disease and dementia with Lewy bodies: a prospective study. Brain. 2019;142: 2051-67.

24. Arora S, Baig F, Lo C, Barber TR, Lawton MA, Zhan A, et al. Smartphone motor testing to distinguish idiopathic REM sleep behavior disorder, controls, and PD. Neurology. 2018;91:e1528-38 The authors demonstrate the ability for smartphone tests to discriminat between PD, healthy controls, and idiopathic REM sleep behavior disorder with a high degree of accuracy.

25. Singh S, Xu W. Robust detection of Parkinson's disease using harvested smartphone voice data: a telemedicine approach. Telemed J E Health. 2020;26:327-34.

26. Zhang YN. Can a smartphone diagnose Parkinson disease? A deep neural network method and telediagnosis system implementation. Parkinsons Dis. 2017;2017:6209703.

27. Rusz J, Hlavnicka J, Tykalova T, Novotny M, Dusek P, Sonka K, et al. Smartphone allows capture of speech abnormalities associated with high risk of developing Parkinson's disease. IEEE Trans Neural Syst Rehabil Eng. 2018;26:1495-507.

28. Del Din S, Elshehabi M, Galna B, Hobert MA, Warmerdam E, Suenkel U, et al. Gait analysis with wearables predicts conversion to Parkinson disease. Ann Neurol. 2019;86:357-67 In a large cohort of older adults, the authors identified wearable sensor-derived gait features that predicted PD diagnosis.

29. von Coelln R, Dawe RJ, Leurgans SE, Curran TA, Truty T, Yu L, et al. Quantitative mobility metrics from a wearable sensor predict incident parkinsonism in older adults. Parkinsonism Relat Disord. 2019;65:190-6.

30. Lalvay L, Lara M, Mora A, Alarcon F, Fraga M, Pancorbo J, et al. Quantitative measurement of akinesia in Parkinson's disease. Mov Disord Clin Pract. 2017:4:316-22.

31. Ellis RJ, Ng YS, Zhu S, Tan DM, Anderson B, Schlaug G, et al. A validated smartphone-based assessment of gait and gait variability in Parkinson's disease. PLoS One. 2015;10:e0141694.

32. Fiems CL, Miller SA, Buchanan N, Knowles E, Larson E, Snow R, et al. Does a sway-based mobile application predict future falls in people with Parkinson disease? Arch Phys Med Rehabil. 2020;101: 472-8.

33. Kim HB, Lee HJ, Lee WW, Kim SK, Jeon HS, Park HY, et al. Validation of freezing-of-gait monitoring using smartphone. Telemed J E Health. 2018;24:899-907.

34. Yahalom G, Yekutieli Z, Israeli-Korn S, Elincx-Benizri S, Livneh V, Fay-Karmon T, et al. Smartphone based timed up and go test can identify postural instability in Parkinson's disease. Isr Med Assoc J. 2020;22:37-42.

35. Hobert MA, Nussbaum S, Heger T, Berg D, Maetzler W, Heinzel S. Progressive gait deficits in Parkinson's disease: a wearable-based biannual 5-year prospective study. Front Aging Neurosci. 2019;11: 22.

36. Suppa A, Kita A, Leodori G, Zampogna A, Nicolini E, Lorenzi P, et al. 1-DOPA and freezing of gait in Parkinson's disease: objective assessment through a wearable wireless system. Front Neurol. 2017;8:406.

37. Haji Ghassemi N, Hannink J, Roth N, Gaßner H, Marxreiter F, Klucken J, Eskofier BM (2019) Turning analysis during standardized test using on-shoe wearable sensors in Parkinson's disease. Sensors (Basel, Switzerland) 19, 3103.

38. Silva de Lima AL, Smits T, Darweesh SKL, Valenti G, Milosevic M, Pijl M, et al. Home-based monitoring of falls using wearable sensors in Parkinson's disease. Mov Disord. 2020;35:109-15.

39. Pickering RM, Grimbergen YA, Rigney U, Ashburn A, Mazibrada $\mathrm{G}$, Wood B, et al. A meta-analysis of six prospective studies of falling in Parkinson's disease. Mov Disord. 2007;22:1892-900.

40. Lo C, Arora S, Baig F, Lawton MA, El Mouden C, Barber TR, et al. Predicting motor, cognitive \& functional impairment in Parkinson's. Ann Clin Transl Neurol. 2019;6:1498-509 The authors demonstrate that performance on smartphone tests can predict key clinical milestones at 18 months.

41. Jeon H, Lee W, Park H, Lee HJ, Kim SK, Kim HB, et al. Highaccuracy automatic classification of Parkinsonian tremor severity using machine learning method. Physiol Meas. 2017;38:1980-99.

42. López-Blanco R, Velasco MA, Méndez-Guerrero A, Romero JP, Del Castillo MD, Serrano JI, et al. Smartwatch for the analysis of rest tremor in patients with Parkinson's disease. J Neurol Sci. 2019;401:37-42.

43. Gatsios D, Antonini A, Gentile G, Marcante A, Pellicano C, Macchiusi L, et al. Feasibility and utility of mHealth for the remote monitoring of parkinson disease: randomized controlled trial. JMIR Mhealth Uhealth. 2020.

44. Lee CY, Kang SJ, Hong SK, Ma HI, Lee U, Kim YJ. A validation study of a smartphone-based finger tapping application for quantitative assessment of bradykinesia in Parkinson's disease. PLoS One. 2016;11:e0158852.

45. Printy BP, Renken LM, Herrmann JP, Lee I, Johnson B, Knight E, et al. Smartphone application for classification of motor impairment 
severity in Parkinson's disease. Conf Proc IEEE Eng Med Biol Soc. 2014;2014:2686-9.

46. Artusi CA, Mishra M, Latimer P, Vizcarra JA, Lopiano L, Maetzler $\mathrm{W}$, et al. Integration of technology-based outcome measures in clinical trials of Parkinson and other neurodegenerative diseases. Parkinsonism Relat Disord. 2018;46(Suppl 1):S53-6.

47. Zhan A, Mohan S, Tarolli C, Schneider RB, Adams JL, Sharma S, et al. Using smartphones and machine learning to quantify Parkinson disease severity: the mobile Parkinson disease score. JAMA Neurol. 2018;75:876-80.

48. Mantri S, Wood S, Duda JE, Morley JF. Comparing self-reported and objective monitoring of physical activity in Parkinson disease. Parkinsonism Relat Disord. 2019;67:56-9.

49. Galperin I, Hillel I, Del Din S, Bekkers EMJ, Nieuwboer A, Abbruzzese G, et al. Associations between daily-living physical activity and laboratory-based assessments of motor severity in patients with falls and Parkinson's disease. Parkinsonism Relat Disord. 2019;62:85-90.

50. Mirelman A, Hillel I, Rochester L, Del Din S, Bloem BR, Avanzino $\mathrm{L}$, et al. Tossing and turning in bed: nocturnal movements in Parkinson's disease. Mov Disord. 2020; The authors demonstrated that wearable devices can quanitfy nocturnal movements in PD and detect differences based on disease stage.

51. Arroyo-Gallego T, Ledesma-Carbayo MJ, Sanchez-Ferro A, Butterworth I, Mendoza CS, Matarazzo M, et al. Detection of motor impairment in Parkinson's disease via mobile touchscreen typing. IEEE Trans Biomed Eng. 2017;64:1994-2002.

52. Iakovakis D, Diniz JA, Trivedi D, Chaudhuri RK, Hadjileontiadis LJ, Hadjidimitriou S, et al. Early Parkinson's disease detection via touchscreen typing analysis using convolutional neural networks. Conf Proc IEEE Eng Med Biol Soc. 2019;2019:3535-8.

53. Iakovakis D, Hadjidimitriou S, Charisis V, Bostantzopoulou S, Katsarou Z, Hadjileontiadis LJ. Touchscreen typing-pattern analysis for detecting fine motor skills decline in early-stage Parkinson's disease. Sci Rep. 2018;8:7663.

54. Liddle J, Ireland D, McBride SJ, Brauer SG, Hall LM, Ding H, et al. Measuring the lifespace of people with Parkinson's disease using smartphones: proof of principle. JMIR Mhealth Uhealth. 2014;2:e13.

55. Liddle J, Sundraraj A, Ireland D, Bennett S, Stillerova T, Silburn P. Impact of deep brain stimulation on people with Parkinson's disease: a mixed methods feasibility study exploring lifespace and community outcomes. Hong Kong J Occup Ther. 2019;32:97-107.

56. Antonini A, Gentile G, Giglio M, Marcante A, Gage H, MML T, et al. Acceptability to patients, carers and clinicians of an mHealth platform for the management of Parkinson's disease (PD_Manager): study protocol for a pilot randomised controlled trial. Trials. 2018;19:492.

57. Hssayeni MD, Burack MA, Jimenez-Shahed J, Ghoraani B. Assessment of response to medication in individuals with Parkinson's disease. Med Eng Phys. 2019;67:33-43.

58. Vizcarra JA, Sanchez-Ferro A, Maetzler W, Marsili L, Zavala L, Lang AE, et al. The Parkinson's disease e-diary: developing a clinical and research tool for the digital age. Mov Disord. 2019;34:676-81.

59. Lopane G, Mellone S, Corzani M, Chiari L, Cortelli P, CalandraBuonaura G, et al. Supervised versus unsupervised technologybased levodopa monitoring in Parkinson's disease: an intrasubject comparison. J Neurol. 2018;265:1343-52.

60. Lee W, Evans AH, Williams DR. Objective measurement and characterization of sleep benefit in Parkinson's disease. Mov Disord Clin Pract. 2017;4:590-6.

61. Isaacson $\mathrm{SH}$, Boroojerdi $\mathrm{B}$, Waln $\mathrm{O}, \mathrm{McGraw} \mathrm{M}$, Kreitzman DL, Klos K, et al. Effect of using a wearable device on clinical decisionmaking and motor symptoms in patients with Parkinson's disease starting transdermal rotigotine patch: a pilot study. Parkinsonism Relat Disord. 2019;64:132-7.
62. Joshi R, Bronstein JM, Keener A, Alcazar J, Yang DD, Joshi M, et al. PKG movement recording system use shows promise in routine clinical care of patients with Parkinson's disease. Front Neurol. 2019;10:1027.

63. Elm JJ, Daeschler M, Bataille L, Schneider R, Amara A, Espay AJ, et al. Feasibility and utility of a clinician dashboard from wearable and mobile application Parkinson's disease data. NPJ Digit Med. 2019;2:95

64. Riggare S, Hagglund M. Precision medicine in Parkinson's disease - exploring patient-initiated self-tracking. J Parkinsons Dis. 2018;8: 441-6.

65. Lakshminarayana R, Wang D, Burn D, Chaudhuri KR, Galtrey C, Guzman NV, et al. Using a smartphone-based self-management platform to support medication adherence and clinical consultation in Parkinson's disease. NPJ Parkinsons Dis. 2017;3:2.

66. Horin AP, McNeely ME, Harrison EC, Myers PS, Sutter EN, Rawson KS, et al. Usability of a daily mHealth application designed to address mobility, speech and dexterity in Parkinson's disease. Neurodegener Dis Manag. 2019;9:97-105.

67. Fung A, Lai EC, Lee BC. Usability and validation of the smarter balance system: an unsupervised dynamic balance exercises system for individuals with Parkinson's disease. IEEE Trans Neural Syst Rehabil Eng. 2018;26:798-806.

68. Balachandar A, Fasano A. Characterizing orthostatic tremor using a smartphone application. Tremor Other Hyperkinet Mov (N Y). 2017;7:488.

69. Chien JH, Torres-Russotto D, Wang Z, Gui C, Whitney D, Siu KC. The use of smartphone in measuring stance and gait patterns in patients with orthostatic tremor. PLoS One. 2019;14:e0220012.

70. Yahalom H, Israeli-Korn S, Linder M, Yekutieli Z, Karlinsky KT, Rubel Y, et al. Psychiatric patients on neuroleptics: evaluation of parkinsonism and quantified assessment of gait. Clin Neuropharmacol. 2020;43:1-6.

71. Gordon MF, Grachev ID, Mazeh I, Dolan Y, Reilmann R, Loupe PS, et al. Quantification of motor function in Huntington disease patients using wearable sensor devices. Digit Biomark. 2019;3: 103-15.

72. Adams JL, Waddell EM, Dinesh K, Spear KL, Tarolli CG, Elson MJ, et al. GEORGE® - The first smartphone application for Huntington disease: a pilot study (4467). Neurology. 2020;94(15 supplement):4467.

73. Purcell NL, Goldman JG, Ouyang B, Bernard B, O'Keefe JA. The effects of dual-task cognitive interference and environmental challenges on balance in Huntington's disease. Mov Disord Clin Pract. 2019;6:202-12.

74. Kegelmeyer DA, Kostyk SK, Fritz NE, Fiumedora MM, Chaudhari A, Palettas M, et al. Quantitative biomechanical assessment of trunk control in Huntington's disease reveals more impairment in static than dynamic tasks. J Neurol Sci. 2017;376:29-34.

75. Dinesh K, Snyder CW, Xiong M, Tarolli CG, Sharma S, Dorsey ER, et al. A longitudinal wearable sensor study in Huntington's disease. Journal of Huntington's Disease. 2020;9:69-81.

76. Nguyen N, Phan D, Pathirana PN, Horne M, Power L, Szmulewicz D. Quantification of axial abnormality due to cerebellar ataxia with inertial measurements. Sensors (Basel, Switzerland). 2018;18:2791.

77. Caliandro P, Conte C, Iacovelli C, Tatarelli A, Castiglia SF, Reale $\mathrm{G}$, et al. Exploring risk of falls and dynamic unbalance in cerebellar ataxia by inertial sensor assessment. Sensors (Basel, Switzerland). 2019;19:5571.

78. López-Blanco R, Velasco MA, Méndez-Guerrero A, Romero JP, del Castillo MD, Serrano JI, et al. Essential tremor quantification based on the combined use of a smartphone and a smartwatch: the NetMD study. J Neurosci Methods. 2018;303:95-102.

79. Zheng X, Vieira Campos A, Ordieres-Meré J, Balseiro J, Labrador Marcos S, Aladro Y. Continuous monitoring of essential tremor 
using a portable system based on smartwatch. Front Neurol. 2017;8:96.

80. Krack P, Batir A, Van Blercom N, Chabardes S, Fraix V, Ardouin $\mathrm{C}$, et al. Five-year follow-up of bilateral stimulation of the subthalamic nucleus in advanced Parkinson's disease. N Engl J Med. 2003;349:1925-34.

81. Schuepbach WM, Rau J, Knudsen K, Volkmann J, Krack P, Timmermann L, et al. Neurostimulation for Parkinson's disease with early motor complications. N Engl J Med. 2013;368:610-22.

82. Benabid AL, Pollak P, Gervason C, Hoffmann D, Gao DM, Hommel M, et al. Long-term suppression of tremor by chronic stimulation of the ventral intermediate thalamic nucleus. Lancet. 1991;337:403-6.

83. Moro E, LeReun C, Krauss JK, Albanese A, Lin JP, Walleser Autiero $\mathrm{S}$, et al. Efficacy of pallidal stimulation in isolated dystonia: a systematic review and meta-analysis. Eur J Neurol. 2017;24:552-60.

84. Heldman DA, Giuffrida JP, Cubo E. Wearable sensors for advanced therapy referral in Parkinson's disease. J Parkinsons Dis. 2016;6:631-8.

85. Lieber B, Taylor BE, Appelboom G, McKhann G, Connolly ES Jr. Motion sensors to assess and monitor medical and surgical management of Parkinson disease. World Neurosurg. 2015;84:561-6.

86. Chockalingam A, Boggs H, Prusik J, Ramirez-Zamora A, Feustel $\mathrm{P}$, Belasen A, et al. Evaluation of quantitative measurement techniques for head tremor with thalamic deep brain stimulation. Neuromodulation. 2017;20:464-70.

87. Wang J, Gong D, Zhang W, Zhang H, Wang S. Quantifying the influence of DBS surgery in patients with Parkinson's disease during perioperative period by wearable sensors. Conf Proc IEEE Eng Med Biol Soc. 2019;2019:3311-4.

88. Lopes EM, Vilas-Boas MDC, Dias D, Rosas MJ, Vaz R, Cunha JPS. iHandU: a novel quantitative wrist rigidity evaluation device for deep brain stimulation surgery. Sensors (Basel). 2020;20:331.

89. Swann NC, de Hemptinne C, Thompson MC, Miocinovic S, Miller AM, Gilron R, et al. Adaptive deep brain stimulation for Parkinson's disease using motor cortex sensing. J Neural Eng. 2018;15:046006.

90. Herron JA, Thompson MC, Brown T, Chizeck HJ, Ojemann JG, Ko AL. Chronic electrocorticography for sensing movement intention and closed-loop deep brain stimulation with wearable sensors in an essential tremor patient. J Neurosurg. 2017;127:580-7.

91. Arlotti M, Marceglia S, Foffani G, Volkmann J, Lozano AM, Moro E, et al. Eight-hours adaptive deep brain stimulation in patients with Parkinson disease. Neurology. 2018;90:e971-6.

92. Pina-Fuentes D, Little S, Oterdoom M, Neal S, Pogosyan A, Tijssen MAJ, et al. Adaptive DBS in a Parkinson's patient with chronically implanted DBS: a proof of principle. Mov Disord. 2017;32:1253-4.

93. Little S, Tripoliti E, Beudel M, Pogosyan A, Cagnan H, Herz D, et al. Adaptive deep brain stimulation for Parkinson's disease demonstrates reduced speech side effects compared to conventional stimulation in the acute setting. J Neurol Neurosurg Psychiatry. 2016;87:1388-9.

94. Tinkhauser G, Pogosyan A, Tan H, Herz DM, Kuhn AA, Brown P. Beta burst dynamics in Parkinson's disease OFF and ON dopaminergic medication. Brain. 2017;140:2968-81.

95. Rosa M, Arlotti M, Marceglia S, Cogiamanian F, Ardolino G, Fonzo AD, et al. Adaptive deep brain stimulation controls levodopa-induced side effects in Parkinsonian patients. Mov Disord. 2017;32:628-9.

96. Piña-Fuentes D, Beudel M, Van Zijl JC, Van Egmond ME, Oterdoom DLM, Van Dijk JMC, et al. Low-frequency oscillation suppression in dystonia: implications for adaptive deep brain stimulation. Parkinsonism Relat Disord. 2020;79:105-9.
97. Liu Z, Liu Y, Wan X, Yang Y, Wang L, Dou W, et al. Pallidal deep brain stimulation in patients with chorea-acanthocytosis. Neuromodulation. 2018;21:741-7.

98. Fasano A, Mancini M. Wearable-based mobility monitoring: the long road ahead. Lancet Neurol. 2020;19:378-9.

99. Warmerdam E, Hausdorff JM, Atrsaei A, Zhou Y, Mirelman A, Aminian $\mathrm{K}$, et al. Long-term unsupervised mobility assessment in movement disorders. Lancet Neurol. 2020;19:462-70.

100. Martinez-Martin P, Rodriguez-Blazquez C, Kurtis MM, Chaudhuri KR, Group NV. The impact of non-motor symptoms on health-related quality of life of patients with Parkinson's disease. Mov Disord. 2011;26:399-406.

101. Santos-Garcia D, de la Fuente-Fernandez R. Impact of non-motor symptoms on health-related and perceived quality of life in Parkinson's disease. J Neurol Sci. 2013;332:136-40.

102. Prakash KM, Nadkarni NV, Lye WK, Yong MH, Tan EK. The impact of non-motor symptoms on the quality of life of Parkinson's disease patients: a longitudinal study. Eur J Neurol. 2016;23:854-60.

103. Center PR, Mobile Fact Sheet,Pew Research Center, https://www. pewresearch.org/internet/fact-sheet/mobile/,

104. Silver L, Smartphone Ownership Is Growing Rapidly Around the World, but Not Always Equally,Pew Research Center, https:// www.pewresearch.org/global/2019/02/05/smartphoneownership-is-growing-rapidly-around-the-world-but-not-alwaysequally/,

105. Norris P (2001) Digital Divide: Civic Engagement, Information Poverty, and the Internet Worldwide, Cambridge University Press, Cambridge.

106. Cohen S, Waks Z, Elm JJ, Gordon MF, Grachev ID, Navon-Perry $\mathrm{L}$, et al. Characterizing patient compliance over six months in remote digital trials of Parkinson's and Huntington disease. BMC Med Inform Decis Mak. 2018;18:138.

107. Fan X, Wang D, Hellman B, Janssen MF, Bakker G, Coghlan R, et al. Assessment of health-related quality of life between people with Parkinson's disease and non-Parkinson's: using data drawn from the ' 100 for Parkinson's' smartphone-based prospective study. Int J Environ Res Public Health. 2018;15.

108. Schneider RB AS, Myers T, Kayson E, Omberg L, Tarolli C, Daeschler M, Macklin E, Dorsey ER, Mangravite L, Schwarzschild M, Simuni T (2019) in International Congress of Parkinson's Disease and Movement Disorders Nice, France.

109. Malekmohammadi M, Shahriari Y, AuYong N, O'Keeffe A, Bordelon Y, Hu X, et al. Pallidal stimulation in Parkinson disease differentially modulates local and network beta activity. J Neural Eng. 2018;15:056016.

110. Hell F, Plate A, Mehrkens JH, Botzel K. Subthalamic oscillatory activity and connectivity during gait in Parkinson's disease. Neuroimage Clin. 2018;19:396-405.

111. Canessa A, Pozzi NG, Arnulfo G, Brumberg J, Reich MM, Pezzoli G, et al. Striatal dopaminergic innervation regulates subthalamic beta-oscillations and cortical-subcortical coupling during movements: preliminary evidence in subjects with Parkinson's disease. Front Hum Neurosci. 2016;10:611.

112. Panov F, Levin E, de Hemptinne C, Swann NC, Qasim S, Miocinovic S, et al. Intraoperative electrocorticography for physiological research in movement disorders: principles and experience in 200 cases. J Neurosurg. 2017;126:122-31.

113. Dorsey ER, Omberg L, Waddell E, Adams JL, Adams R, Ali MR, et al. Deep phenotyping of Parkinson's disease. J Parkinsons Dis. $2020 ; 10: 855-73$

Publisher's Note Springer Nature remains neutral with regard to jurisdictional claims in published maps and institutional affiliations. 